\title{
Acceptable Intrusiveness of Online Help in Mobile Devices
}

\author{
Ohad Inbar, Talia Lavie, Joachim Meyer \\ Department of Industrial Engineering and Management and T-Labs @ BGU \\ Ben-Gurion University of the Negev, Beer Sheva 84105, Israel \\ +97286472216 \\ (inbaro, tlavie, joachim)@bgu.ac.il
}

\begin{abstract}
The aim of this study was to examine how users perceive help on a mobile device with respect to the presentation format and the severity of the scenario the user encounters. We examined how 92 participants responded to four different formats of help offered for five different scenarios varying in their severity level using static images displayed in a web browser. The intrusiveness of the help format and the severity of the scenario affected participants' interest in receiving help. In certain conditions it may be helpful to suggest help, in particular when users do not know how to ask for it but show interest in receiving it when offered.
\end{abstract}

\section{Categories and Subject Descriptors}

H.5.2 [Information Interfaces and Presentation]: User Interfaces---User-centered design, Interaction styles; J.7 [Computers in Other Systems]: Consumer products

\section{General Terms}

Design, Experimentation, Human Factors.

\section{Keywords}

Mobile devices, help format, mobile assistance.

\section{INTRODUCTION}

Mobile phones, especially "smart phones", continuously gain functionality and features. A familiar side-effect of increased utility is a decrease in usability and user-experience [1]. A report by WDSglobal [7] claims that in the United Kingdom 1 in 7 cell phones is returned within the first year of purchase and claimed to be "faulty". Examining the reasons for return show that $63 \%$ had actually no hardware or software fault. Rather, a major reason for return $(24 \%)$ was reported to be "abandoning the device after a lengthy and frustrating battle with the usability of functions or applications". An additional $31 \%$ of those returning phones had problems configuring and changing settings on their phones. The cost of these 'no fault found' returns to the industry is enormous over 4 billion dollars worldwide.

One way to deal with the ever-increasing functionality is by assisting people in using their devices. Effective assistance will allow users to acquire the new skills and knowledge they need to easily operate an unfamiliar device or function.

Copyright is held by the author/owner(s). MobileHCI'09, September 15 - 18, 2009, Bonn, Germany. ACM 978-1-60558-281-8.

\subsection{Seeking Help}

Previous research has shown that people do not effectively seek help [4], and that, specifically in the context of computer systems, users generally view help as a last resort, which they would rather avoid [5]. Moreover, people rarely use documentation, whether printed or online [1], [8] and show a significant preference for asking others, including help desk and colleagues, over using documentation [4]. Willis [9] suggests a model of human helpseeking behavior in an application environment consisting of five steps of seeking help, the first of which is becoming aware of the need for help. This awareness typically arises when a person starts to use a new system or function or encounters a problem. Inefficient working methods, on the other hand, may go unnoticed [4], leaving people unaware of benefits help could provide.

\subsection{Approaching the User}

How users respond to assistance offered by the system has seldom been examined. The few studies that did examine user response related to a specific help system (e.g., Microsoft's 'clippy'). Our aim is to evaluate responses in a more general perspective. As notification systems are very similar to system-initiated assistance, we reviewed relevant literature, with the goal of gaining insight as to how to best offer assistance.

We found that deciding on the optimal presentation mode and timing depends on two groups of factors: (1) contextual factors, i.e., the user's state and the usage conditions, and (2) the value of the information provided in the notification. An obvious disadvantage of notifications is their distractive effect, which may interfere with performance of the current task. [2] summarized eleven factors that impact the perceived burden of an interruption. These can be grouped into five categories related to the user, the notification itself, the task performed following the notification, the user activities and social factors.

\subsection{The Mobile Context}

Assisting users of mobile devices is a complex challenge. Attentional limitations, resulting from the unique context of mobile use, lead to an impulsive, fragmented and drastically short attention span in mobile interaction [6]. Apparently, users allocate just 4 to 8 seconds of attention to their devices at a time.

Two implications for presenting help on mobile devices arise from these findings: First - it is important to ensure the user's attention to help-information in situations in which important decisions have to be made. One example is a salient visual message describing the situation and potential actions. Second - when the problem is minor, it is preferable not to divert excessive 
attentional resources from other activities. In this case, a small visual cue could indicate that further assistance is available.

\subsection{About this Study}

This study examines users' perception of help provided to mobile devices as a function of the presentation format and the severity of the scenario encountered. Given people's attitudes towards assistance and notifications, as reviewed in section 1.1, our aim was to examine when people would want to receive help in their mobile handset. We hypothesized that people's willingness would depend on the severity of the scenario they face and the format in which the help is presented. We assumed people would show more interest in help for more severe scenarios and less interest in help formats perceived to be more intrusive.

\section{METHOD}

\subsection{Preliminary Survey - User Scenarios}

In order to evaluate assistance formats on scenarios for which users would actually want help, we conducted a preliminary survey to assess subjective evaluations of the perceived severity of various usage scenarios. In addition, we asked about the perceived importance of receiving help on the issues and the degree to which respondents believed help could be beneficial.

From an initial 23 scenarios of various phone functions (including a specific email application - "Email Pro" - available on Deutsche Telekom phones in Germany) we eventually chose 15 to include in the preliminary survey. These were presented in a questionnaire that was distributed to a convenience sample of 13 respondents, all highly experienced with computers and mobile devices. No item received very low rankings (below 3 on a seven-point scale) but some were clearly considered more severe.

Based on the results of this survey, we decided to include the following five scenarios in the experiment:

1. Synchronization: exchange of data between the phone and a PC, with possible loss of data

2. Offline: writing an email while in 'offline' mode

3. Battery: improving battery life by reducing the frequency of checking for new emails

4. cc: informing the user about easier ways for using the cc ('carbon copy') function

5. MMS: informing the user about the ability to send pictures (as MMS) immediately after taking them

\subsection{Online Experimental System}

The experiment was conducted with an online experiment system, developed with a PHP platform and run on a private server. Participants received an invitation to participate in the experiment on their own computer. The introduction to the experiment stated the overall goal of the experiment, the compensation ( 3 prizes of 200NIS each to be raffled among those who completed the experiment, independent of performance), and some basic instructions. Users were then asked for personal details, including prior experience with mobile services. Each survey consisted of all five scenarios, each presented with four different formats (described in section 2.4), meaning each participant received all 20 items, each on a separate page.

\subsection{Participants}

Participants were recruited using the university online forum and student friends within the university. Overall, 92 participants (40 males and 52 females) completed the online experiment. Sixtytwo participants were between 19-29 years old. Over half (49 of 92) had a high school diploma or some college education, with the remainder having a BA or higher level of education. When asked for their experience with advanced mobile services, most participants stated relatively little experience with mobile email, web browsing and MMS (multimedia messaging).

\subsection{Assistance Formats}

Four assistance formats, designed according to the amount of assistance information initially presented to the user, were used in this study. The "icon" format consists of a small icon displayed in the corner of the screen (Figure 1). Clicking this icon opens a fullscreen help dialog that is later closed by the user. The two "Bubble" formats - the "Self-closing bubble" and the "Manual bubble" (Figure 2) provide more initial information, but they are potentially more intrusive. Visually both bubble formats are identical, presenting a brief summary of the assistance information at the bottom of the screen. Clicking the bubble opens the same full help screen as in the "Icon" format. The "Manual" bubble requires the user to manually close it and the "Self closing" bubble closes by itself after a few seconds (when not pressed). The "Full help" screen holds the most information and appears automatically (based on the system's logic). It is not preceded by an icon or a bubble (Figure 3 ).

\subsection{Information Gathered}

Each of the formats (for each of the scenarios) discussed in section 2.4 was displayed to each user and was followed by four 7-point scale questions regarding the helpfulness and the intrusiveness of the assistance. In order to validate the rating found in the preliminary survey, we also asked participants to rate the severity and importance of each scenario. These questions were presented at the end of the survey, together with a brief summary of each scenario.
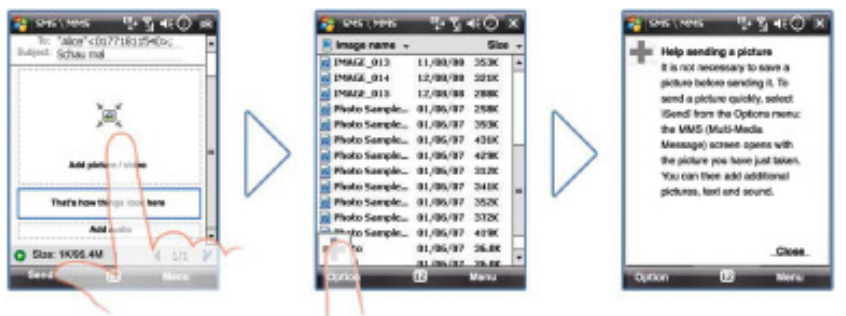

Figure 1 - "Icon" format
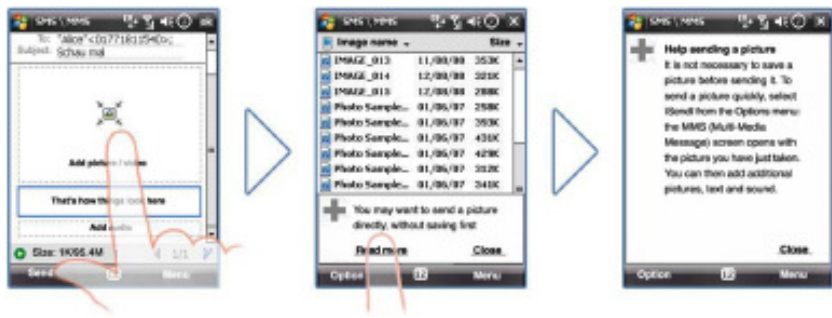

Figure 2 - "Bubble" formats 


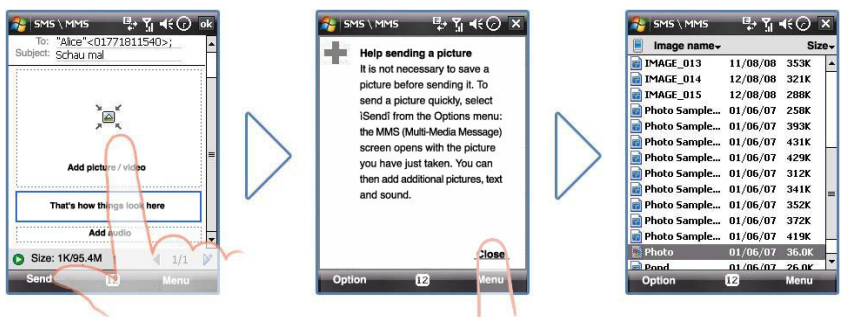

Figure 3 - Full help screen

\section{RESULTS}

Two-way ANOVAs with repeated measures were performed on all the questions in this study, with the scenario and help format as the repeated measure variables. No significant effects were found for either gender or age, for all parameters in this study.

\subsection{Significance of Help}

We first analyzed participants' perceived severity of the different scenarios. Significant differences were found between the perceived severity levels of the scenarios, $F(4,348)=63.439$, $\mathrm{p}<0.01$. While the MMS and cc scenarios were not perceived to be severe (mean ratings of 2.9 and 3 respectively), the battery and offline, and especially the synchronization scenario, were perceived as more severe (mean ratings of 4.6, 4.8, and 5.73 respectively). The results of the perceived severity level were highly correlated to those in the preliminary study $(r=0.95)$ demonstrating high validity of these scenarios.

Participants were also asked to indicate the importance of receiving help for each of the usability scenarios. Help was perceived to be significantly more important for the more severe scenarios, $\mathrm{F}(4,348)=60.7, \mathrm{p}<0.01$ (mean ratings of cc -3.4 , MMS - 3.6, Battery - 4.8, Offline - 5.3, and Synchronization - 5.9). As expected, very high correlations were found between the perceived severities of the scenario and the perceived importance of receiving help $(\mathrm{r}=0.99)$.

\subsection{Intrusiveness of Help}

Participants perceived the help formats as having different levels of intrusiveness, $\mathrm{F}(3,255)=53, \mathrm{p}<0.01$. "Full screen" was perceived to be the most intrusive (mean rating 4.4) followed by the two "bubble" formats (mean ratings 3.3). The "icon" format was perceived to be least intrusive (mean rating 2.5).

A significant interaction help format $*$ scenario was also found, $\mathrm{F}(12,1020)=3.8, \mathrm{p}<0.01$. As seen in Figure 4, help in the format of an icon was perceived to be least intrusive for all scenarios, and help offered in the full screen format was perceived to be most intrusive for all scenarios. Nevertheless, planned comparisons examining the differences among the scenarios in the full screen format revealed significant differences between the scenarios, $\mathrm{F}(4,340)=506.6, \mathrm{p}<0.01$. The perceived intrusiveness of the help decreased with the severity of the scenario, being lowest for the Synchronization scenario.

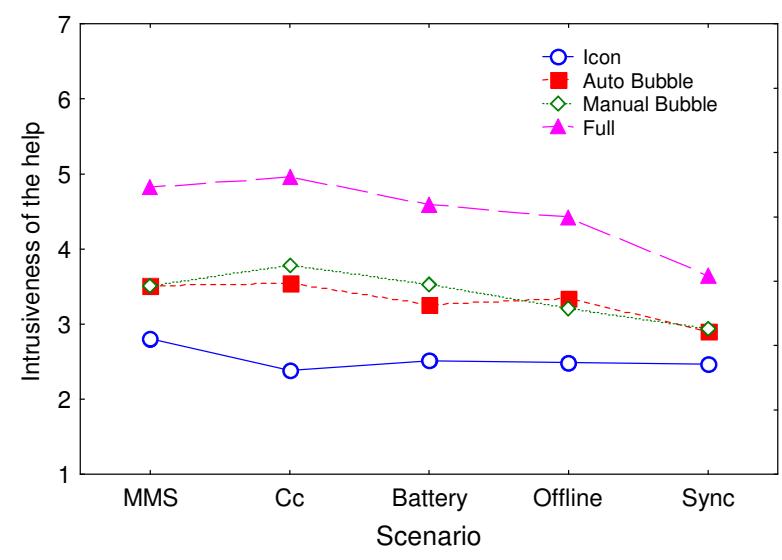

Figure 4 - Mean ratings of the perceived intrusiveness of the help for the different scenarios

\subsection{Interest in Receiving Help}

Overall, participants indicated interest in receiving help in their mobile handset, although level of interest depended on the help format, $F(3,252)=12.09, p<0.01$. The "bubble" formats received the highest ratings (manual bubble - mean rating 4.8, self-closing bubble - mean rating 4.7), followed by the "icon" format (mean rating 4.3). The "full screen" format received the lowest ratings (mean rating 4), particularly for the less severe scenarios.

Planned comparisons showed significantly lower interest rating for the full screen format in the MMS $F(1,84)=25.3, p<0.01, \mathrm{Cc}$ $\mathrm{F}(1,84)=29.5, \quad \mathrm{p}<0.01$ and Battery $\mathrm{F}(1,84)=21.8, \quad \mathrm{p}<0.01$ scenarios. For more severe scenarios (Offline and, especially, Synchronization), participants did show a greater interest in receiving full screen help than help in other formats. Opposite findings were found for help in the format of an icon. Here, participants indicated less interest in this format for severe scenarios - Offline $\mathrm{F}(3,252)=12.8 \quad \mathrm{p}<0.01$ and Sync $\mathrm{F}(3,252)=10.67, \mathrm{p}<0.01$.

Interest in receiving help also varied significantly between the scenarios themselves, $\mathrm{F}(12,1008)=7.65, \mathrm{p}<0.01$. As presented in Figure 5, the severity of the scenario, along with the specific help format, affected interest levels.

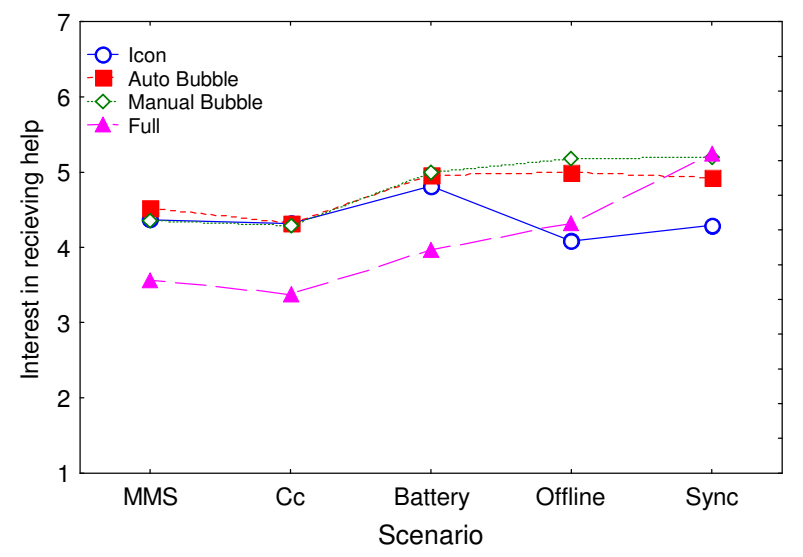

Figure 5 - Mean ratings of the participants' interest level in receiving help for the different scenarios 


\section{DISCUSSION}

Research on cell phones suggests that users can benefit from receiving assistance for operating their handset and using its various applications [6]. Nevertheless, previous research has shown that users don't seek help effectively [3], and they prefer to request assistance from people. System-initiated help may be one solution, though it also has a downside, as users may view it as interruptive. Research has not yet studied aspects of receiving assistance in mobile applications, in which the issue is even more complex than that of receiving help for PC applications due to, among other things, the small screen size and the greatly varying context of use.

Our aim was to examine people's interest in receiving such help. The conditions we examined relate to the severity of the scenarios and the format of the help, in terms of the amount of information initially displayed, assumingly affecting its intrusiveness level.

Participants rated scenarios that concerned potential loss of information as being more severe than those that involved inefficient use. Subsequently, they perceived the importance of receiving help as corresponding to the scenario's severity level (i.e., the more severe the scenario, the more important it is to receive help). Overall, participants did show an interest in receiving help. Users might not request help for efficiency scenarios (e.g., MMS and cc scenarios), but they may be willing to accept it when it is offered to them.

We selected four help formats, with three different amounts of initially presented information, assuming that a greater amount of initial information would be perceived as more intrusive. Our results support this hypothesis. We also found that the perceived intrusiveness of help is affected by other factors, such as the severity of the scenarios.

The help format offered affected the participants' interest in receiving help. While in general, they were most interested in the two bubble formats and least interested in the full screen help format, results of the interaction with the scenarios showed that the most influential factor was the severity of the scenarios. This demonstrates that intrusiveness alone is not a determining factor in the willingness to receive help. We postulate that people's near equal level of interest in the two bubble formats may have been a result of the static presentation format.

\section{SUMMARY AND CONCLUSIONS}

Although users frequently need assistance, providing it is a complex matter, especially for mobile applications. It is therefore important to try to offer the help in a way that will best support user needs and minimize its disruptive effect. For instance, more intrusive help may be offered for severe scenarios, while less intrusive help should be offered for scenarios concerning degraded efficiency ramifications. Additionally, since we suggest offering help instead of having the user request it (push vs. pull), the help in our study may be viewed as a type of notification. It may therefore be beneficial to apply insight from the study of notifications. Similar to the eleven factors affecting the perceived burden of an interruption, identified in [2], we can classify factors affecting the perceptions and willingness to accept assistance. Here, too, among other things, the form of assistance, the task being performed and the user's current activities may affect attitudes towards assistance.

This study only addressed the form of assistance offered, but additional aspects should be examined to gain a better understanding of how and when to offer help to best suit users' needs. Furthermore, in order to examine the actual intrusiveness and attitudes towards assistance, it is important to examine attitudes towards assistance when participants are engaged in tasks, either related or not related to the assistance offered, preferably while using actual devices rather than static images.

\section{ACKNOWLEDGMENTS}

Our thanks to the group at FIT who provided us with the usage scenarios and to Deutsche Telekom who funded this research.

\section{REFERENCES}

[1] Ceaparu, I., Lazar, J., Bessiere, K., Robinson, J., Shneiderman, B. (2004). Determining causes and severity of end-user frustration, International Journal of HumanComputer Interaction, 17(3), 333-356.

[2] Ho, J., Intille, S. S. (2005). Using context-aware computing to reduce the perceived burden of interruptions from mobile devices. Human Factors in Computing Systems: Proceedings of $\mathrm{CHI}^{\prime}$ 05, New York: ACM Press, 909-918.

[3] Nelson-Le Gall, S. (1981). Help-seeking: An understudied problem solving skill in children. Developmental Review, 1 , 267-293.

[4] Novick D.G, Ward K. (2006). Why Don't People Read the Manual? Proceedings of the 24th annual ACM international conference on design of communication, $11-18$.

[5] Novick, D., Elizadle, E., Bean, N. (2007). Toward a more accurate view of when and how people seek Help with computer Applications. SIGDOC '07: Proceedings of the 25th annual ACM international conference on design of communication, 95 - 102.

[6] Oulasvirta, A., Tamminen, S., Roto, V., Kuorelahti, J. (2005). Interaction in 4-second bursts: The fragmented nature of attentional resources in mobile HCI, In Proceedings of SIGCHI Conference on Human Factors in Computing Systems, ACM Press, New York, 919-928.

[7] Overton, D. (2006). 'No Fault Found' returns cost the mobile industry $\$ 4.5$ billion per year. http://www.wdsglobal.com/news/whitepapers/20060717/200 60717.asp (accessed January 2009).

[8] Shedroff, N. (1999). Information Interaction Design: A Unified Field Theory of Design. In Information Design, (Ed, Jacobson, R.) MIT Press, 267-293.

[9] Willis, M. (2006). Building effective help systems: Modeling human help seeking behavior. Proceedings of the 20th conference of the computer-human interaction special interest group. 